\title{
LA CONSTITUCIÓN DEL CUERPO PROPIO Y LA DESCRIPCIÓN DE LA CARNE EN LA CRÍTICA HENRIANA A MERLEAU-PONTY
}

\author{
Carlos Daniel Belvedere* \\ doi:10.11144/Javeriana.uph31-63.ccdc
}

\begin{abstract}
RESUMEN
En este trabajo, mi objetivo es dar cuenta de las objeciones de Michel Henry a Merleau-Ponty en lo concerniente a la cuestión del cuerpo propio y la carne. A tales efectos, comienzo por presentar la posición de Merleau-Ponty. Luego introduzco las críticas de Henry, que hacen foco en Fenomenología de la Percepción y Lo visible y lo invisible. A continuación sopeso estas críticas en general y me concentro, en particular, en la significación que tiene para un balance de este debate el último texto de Merleau-Ponty, dedicado a la dióptrica de Descartes. Considero, para finalizar, que este trabajo inconcluso de Merleau-Ponty abre un canal de diálogo más fecundo que el que las obras criticadas por Henry permitían.
\end{abstract}

Palabras clave: cuerpo propio; carne; fenomenología material; Henry; Merleau-Ponty

\footnotetext{
* Consejo Nacional de Investigaciones Científicas y Técnicas (CONICET), Universidad de Buenos Aires y Universidad Nacional de General Sarmiento, Buenos Aires, Argentina. ReCiBIDO: 29.12.13 ACEPTADO: 25.03.14 DiSPONIBLE EN LÍNEA: 02.12.14

Para citar este artículo: Belvedere, C.D. (2014). La construcción del cuerpo propio y la descripción de la carne en la crítica henriana a Merleau-Ponty. Universitas Philosophica, 31(63), pp. 119-142, ISSN 0120-5323, ISSN en línea 2346-2426, doi: 10.11144/Javeriana. uph31-63.ccdc
} 


\title{
THE CONSTITUTION OF THE OWN BODY AND DESCRIPTION OF THE FLESH IN HENRY'S CRITIQUE OF MERLEAU- PONTY
}

\author{
Carlos Daniel Belvedere
}

\begin{abstract}
The aim of this paper is to account for Michel Henry's critique of Merleau-Ponty as regards the own body and the flesh. To that end, I start by displaying Merleau-Ponty's position on these matters. Then I present Henry's critique, which are focused on Phenomenology of Perception and The Visible and the Invisible. After that, I consider these objections in general and concentrate on the meaning of Merleau-Ponty's latest work on Descartes' dioptric, in particular. Finally, I argue that his unfinished work opens more fruitful channels of dialogue than the works criticized by Henry.
\end{abstract}

Key words: own body; flesh; material phenomenology; Henry; Merleau-Ponty 
Nos PROPONEMOS, EN ESTE ARTículo, RESEÑAR una de las polémicas más frontales y significativas de la fenomenología francesa: aquella en la que Michel Henry, de manera enfática y sin concesiones, ha cuestionado a Maurice Merleau-Ponty.

Hay razones de peso para que esta confrontación haya ocurrido. Por un lado, el debate era inevitable, tratándose de dos de los más descollantes fenomenólogos en lengua francesa. Algo debía decir uno de otro, dada esta cercanía de carácter contextual. Por otro lado, la proximidad era poco más que circunstancial. Si bien ambos pensadores convergían temáticamente al ocuparse del cuerpo propio y de la carne como aspectos medulares de sus respectivas filosofias, las divergencias no podían ser mayores en lo que respecta a la manera de concebir estos fenómenos fundamentales. Semejante combinación de afinidad temática y desacuerdo filosófico hacía, entonces, inevitable la confrontación.

Por cuestiones generacionales, es comprensible que Henry haya sido quien debió expresar estas diferencias. Siendo algo más joven que MerleauPonty, y habiéndolo sobrevivido por mucho, era lógico que tomara él la voz cantante a la hora de sincerar sus desacuerdos.

En lo que sigue, intentaremos dar cuenta de lo que bien podríamos llamar "el diferendo" entre Merleau-Ponty y Henry. Evidentemente, por los motivos mencionados, retomaremos principalmente la argumentación del segundo, pues es quien presenta las objeciones del caso. Sin embargo, comenzaremos y concluiremos refiriéndonos al primero, retomando su palabra, en esta ocasión, a nivel expositivo y no polémico. Seremos nosotros, finalmente, quienes lo haremos contrargumentar, en busca de un campo fértil para un diálogo más profundo que el que históricamente tuvo lugar.

Con este objetivo en mente, comenzaremos por reseñar la concepción merleau-pontiana de la carne en el contexto de su recuperación de las descripciones de Husserl sobre el cuerpo propio y de la generalización de lo Sensible que el filósofo francés produce en su última obra. Luego presentaremos las principales objeciones que Henry le hace a dos obras capitales de este: Fenomenología de la percepción y Lo visible y lo invisible. Después volveremos a un trabajo inconcluso de Merleau-Ponty no tenido en cuenta por Henry, donde encontraremos algunos de los desarrollos que aparentemente faltarían en su obra previa; en particular, la tematización de la afectividad como inmanencia. Concluiremos con algunas consideraciones 
personales, muy breves, en las que haremos hincapié en esta desatendida convergencia entre ambos autores, sin por ello descuidar las insalvables distancias que los separan.

\section{Los antecedentes husserlianos}

MerLEAu-Ponty RETOMA La DESCRIPCIÓn hUSSERLIANa de "la incompletud de mi cuerpo" y "su ambigüedad como cuerpo tocante y cuerpo tocado" (Merleau-Ponty, 1999, p. 110) pero, a su vez, la generaliza en dirección a una novedosa filosofía del sintiente en general. A estos efectos, retoma el término alemán Leib, que significa "el cuerpo a la vez viviente y vivido [...] distinto del cuerpo objetivo", y lo traduce como "carne" (Barbaras, 1997, p. 52). Este término es utilizado en sus escritos "en un sentido que excede el del cuerpo propio y que hace de él uno de los conceptos centrales de su ontología" (Barbaras, 1997, p. 52), en la cual es considerado como un "elemento" en el sentido antiguo de "una cosa general" (Barbaras, 1997, p. 60), siendo "a la vez 'noción última' y tejido sub-tendiente al Ser" (Alloa, 2008, p. 79). Comencemos, entonces, presentando los aspectos más significativos de la caracterización husserliana de la constitución del cuerpo propio que han de cimentar los análisis de Merleau-Ponty.

Husserl realiza una magistral descripción de la constitución del cuerpo propio a partir de del ejemplo de las manos que se tocan, el cual será fundamental para la caracterización merelau-pontiana de la carne. En ella muestra que ciertas partes de nuestro cuerpo pueden ser percibidas por el tacto pero no por la vista, por más que haya otras que son a la vez tangibles y visibles (Husserl, 2004, p. 206). En la descripción de este fenómeno, Husserl desarrolla "con una virtuosidad inigualada la tesis aristotélica de que el tocar tiene su privilegio sobre todos los otros sentidos en que para él solo el medio de la percepción es uno con el que percibe, de tal modo que ese sentiente no puede nunca sentir sin sentirse" (Marion, 2005a, p. 61).

En efecto, Husserl considera (en los parágrafos 36 y 37 de Ideas II) que hay una "sorprendente" diferencia de los aparatos táctiles respecto de los visuales. En el primer caso, además del objeto exterior, que se constituye de manera táctil, tenemos un segundo objeto, el cuerpo propio, que se constituye de la misma manera (Husserl, 2004, p. 210). Así es que, en lo táctil podemos experimentar una "doble aprehensión" tal que, la misma 
sensación de tocar, aprehendida en tanto que rasgo característico del objeto "exterior", es también aprehendida en tanto que sensación del objeto-cuerpo propio. Particularmente, en el caso de que una parte de mi cuerpo propio sea a la vez el objeto exterior de otra parte de mi cuerpo, tenemos que las dobles sensaciones y la doble aprehensión conforman un rasgo característico de tal o cual parte del cuerpo propio en tanto que objeto físico. En cambio, nada semejante ocurre con el objeto constituido visualmente (Husserl, 2004 , p. 210). Es por eso que mi cuerpo es la única cosa física que no puedo ver enteramente (García-Baró, 1999, p. 48). Es decir que el ver deja "necesarias lagunas" cuando se trata de nuestro cuerpo físico, que llenamos "constituyendo originariamente en el tacto las partes correspondientes de él. Aunque también, y a la inversa, la percepción del cuerpo físico basada en el tacto comporta vacíos irremediables por el tacto mismo, que sólo palia la vista" (García-Baró, 1999, p. 48)

En breve, la especificidad de la sensación táctil respecto de la sensación puramente visual es que, en la primera, el cuerpo propio se aprehende a sí mismo, simultáneamente, como objeto exterior y como sujeto de la sensación, mientras que nada de esto ocurre en la segunda, pues el ojo no se ve a sí mismo viendo (Husserl, 2004, p. 210). En efecto, no tenemos una cualidad ocular extensiva por la cual un ojo pueda bordear progresivamente al otro como para que se produzca el fenómeno de la doble sensación; ni podemos ver la cosa vista deslizarse sobre el ojo que ve, tocándola continuamente tal como lo podemos hacer, mediante el tacto, con la superficie de nuestra mano (Husserl, 2004, p. 210-211). Precisamente, porque solo hay doble sensación en el tacto es que únicamente allí puede constituirse el cuerpo propio (Husserl, 2004, p. 214).

\section{La posición merleau-pontiana}

Al igual que Husserl, Merleau-Ponty reconoce que mi cuerpo me da sensaciones dobles puesto que, en el paso de una función a otra, puedo reconocer la mano tocada como la misma que toca. Así, "cuando toco mi mano derecha con mi mano izquierda, el objeto mano derecha tiene la singular propiedad de sentir también" (Merleau-Ponty, 1999, p. 109), aunque nunca las dos manos son, a la vez, tocadas y tocantes una respecto de otra. Cuando aprieto mis dos manos una contra otra, no experimento dos sensaciones juntas como si percibiéramos dos objetos yuxtapuestos, sino que percibo 
una organización ambigua en la cual las dos manos pueden alternarse en su función de "tocante" y "tocada" (Merleau-Ponty, 1999, p. 109). En la encarnación de la mano "que lanzo hacia los objetos por explorar",

el cuerpo se sorprende a sí mismo desde el exterior ejerciendo una función de conocimiento, trata de tocarse tocando, esboza 'una suerte de reflexión' y con eso bastaría para distinguirlo de los objetos, de los cuales bien puedo decir que "tocan" mi cuerpo, pero solo cuando está inerte,y sin que jamás los sorprendan en su función exploratoria. (Merleau-Ponty, 1999, p. 109)

Así descrito, el cuerpo se nos muestra como "un objeto afectivo", mientras que "las cosas exteriores solo me son representadas" (MerleauPonty, 1999, p. 109). Esta contraposición nos hace reparar en que "mi cuerpo no se ofrece a la manera de los objetos del sentido externo" y que "estos solo se perfilan sobre el fondo afectivo que lanza originariamente a la conciencia fuera de sí misma" (Merleau-Ponty, 1999, p. 110). En definitiva, el análisis del cuerpo propio nos lo presenta como un objeto-sujeto capaz de ver y de sufrir (Merleau-Ponty, 1999, p. 111).

Sin embargo, a pesar de que la Fenomenología de la percepción muestra una considerable cercanía con Husserl, también presenta una significativa diferencia pues el "tocar" habrá de perder su especificidad, en la medida en que Merleau-Ponty ya no lo contrapone sino que lo articula con el ver. Así, lo que Husserl dice del tocar, Merleau-Ponty lo dirá, indistintamente, del tocar y del ver:

En tanto que ve o toca el mundo, mi cuerpo no puede ser visto ni tocado. Eso mismo que le impide ser jamás un objeto, ser completamente constituido, es aquello mediante lo cual hay objetos. No es ni tangible ni visible en la medida en que es lo que ve y lo que toca. El cuerpo, entonces, no es un objeto exterior cualquiera, que ofreciera solo la particularidad de estar siempre allí. Si es permanente, es de una permanencia absoluta que sirve de fondo a la permanencia relativa de los objetos de eclipse, de los verdaderos objetos. (Merleau-Ponty, 1999, p. 107-108)

Luego, la especificidad del tocar como fenómeno privilegiado para la descripción del cuerpo propio se diluye, equiparándose con la creciente significación que adquiere el ver. No se trata, sin embargo, de una mera equiparación de ver y tocar, sino que, tal como anticipamos al comienzo, Merlau-Ponty nos conduce, con redoblado énfasis en su última e inconclusa 
obra, a una generalización de lo Sensible, en el marco de la cual ver y tocar serían dos de sus expresiones.

En Lo visible y lo invisible, Merleau-Ponty reconduce la glosa de los textos husserlianos en dirección a las problemáticas de su última obra: lo sensible, la carne y lo visible. Además, en la versión merleau-pontiana, la percepción de sí es realizada por un impersonal "ser sensible".

Si mi mano izquierda toca mi mano derecha, y de pronto quiero captar con mi mano derecha el trabajo de mi mano izquierda tocando, esta reflexión del cuerpo sobre sí mismo siempre aborta a último momento: en el momento en que siento mi zurda con mi diestra, en la misma medida ceso de tocar mi mano derecha con mi mano izquierda. Pero este fracaso de último momento no le quita toda verdad a este presentimiento que tenía de poder tocarme tocando: mi cuerpo no percibe, sino que está como construido en torno de la percepción que emerge a través de él; por toda su disposición interna, por sus circuitos sensorio-motores, por las vías de regreso que controlan y relanzan los movimientos, se prepara por así decir para una percepción de sí, incluso si nunca es él lo que percibe ni es él quien lo percibe. (Merleau-Ponty, 1997, p. 24)

La percepción del cuerpo propio, entonces, es hecha por una vida generalizada: "mi mundo privado ha cesado de ser sólo mío, ahora es el instrumento mediante el cual juega otro, la dimensión de una vida generalizada que fue injertada en la mía" (Merleau-Ponty, 1997, p. 27). Por eso, si bien el pensamiento no es exterior (Merleau-Ponty, 1997, p. 52) porque no hay "una relación exterior entre el percipiente y lo percibido" (Merleau-Ponty, 1997, p. 53), tampoco es interior: "la reflexión puede tener la ilusión de ser retorno a sí y de instalarse en la inmanencia, y nuestro poder de volver a nosotros es medido exactamente por un poder de salir de nosotros que no es ni más antiguo ni más reciente que él, y del cual es sinónimo" (Merleau-Ponty, 1997, p. 56). Por eso, no es correcto reemplazar a la percepción por lo percibido ni a lo percibido por la percepción. Semejante movimiento implicaría "reducir la percepción al pensamiento de percibir" -reducción en la que se pierde más de lo que eventualmente se ganaría-; razón por la cual Merleau-Ponty rechaza esta renuncia al mundo para buscar comprenderlo y acceder allí a la incomparable certeza que nos proporciona el "hay" (Merleau-Ponty, 1997, p. 58-59). 
La tesis inmanentista, que Merleau-Ponty rechaza, olvida también la reflexión como acto distinto de retoma en favor de

una suerte de sobrerreflexión que daría cuenta también de ella misma y de los cambios que ella introduciría en el espectáculo, que entonces no perdería de vista la cosa y la percepción brutas, y que finalmente las borraría, no cortaría, por una hipótesis de inexistencia, los lazos orgánicos de la percepción y de la cosas percibidas, y por el contrario, se daría por tarea pensarlas, reflexionar sobre la trascendencia del mundo como trascendencia. (Merleau-Ponty, 1997, p. 61)

Ahora bien, si Merleau-Ponty rechaza el inmanentismo es porque no acepta la oposición de una luz interior y un orden de cosas en sí (MerleauPonty, 1997, p. 67): "como decía Hegel, volver a sí es también salir de sí" (Merleau-Ponty, 1997, p. 74). La noción de carne expresará esta generalidad del ser, ni interior ni exterior, ni visible ni invisible: lo visible del mundo es el "tejido conjuntivo de los horizontes exteriores e interiores", y la "carne ofrecida a la carne" es "generalidad" (Merleau-Ponty, 1997, p. 173n).

Así es como la descripción de la experiencia de las manos que se tocan conduce no solo a la descripción del tocar, sino también a una filosofía de la carne que generaliza esta experiencia, no distinguiendo ya -como lo hacía Husserl - el tocar y el ver. Por esa razón, tras señalar que

existe una relación de principio, un parentesco [...] que solo puede ocurrir si, al mismo tiempo que sentida desde adentro, mi mano es también accesible desde fuera, siendo tangible, por ejemplo, para mi otra mano, si toma lugar entre las cosas que toca, es en un sentido una de ellas, obra finalmente sobre un ser tangible del cual ella también forma parte. (Merleau-Ponty, 1997, p. 176)

Asimismo, "por este entrecruzamiento en ella de lo tocante y lo tangible", los movimientos propios de la mano que toca "se incorporan al universo que interrogan" (Merleau-Ponty, 1997, p. 176). Algo similar ocurre con la visión: no es solo un videre videor sino también un verdadero "tocar del tocar" pues, "cuando mi mano izquierda toca mi mano derecha mientras está palpando las cosas, por la cual el 'sujeto tocante' pasa al rango de tocado, desciende en las cosas, de manera que el tocar se hace en medio del mundo y como en ellas" (Merleau-Ponty, 1997, p. 176). 
En definitiva, Merleau-Ponty (1997, p. 177) nos habla de la unidad de lo sensible gracias a la cual "el espectáculo visible pertenece al tocar ni más ni menos que las "cualidades táctiles". Lo visible y lo tangible son dos "partes totales", dos "cartas completas": "todo visible está tallado en lo tangible, todo ser táctil está prometido de alguna manera a la visibilidad" (MerleauPonty, 1997, p. 177). Así,

hay invasión, abarcamiento, no sólo entre lo tocado y lo tocante, sino también entre lo tangible y lo invisible que está incrustado en él, así como, inversamente, él mismo no es una nada de visibilidad, no es sin existencia visual. Porque el mismo cuerpo ve y toca, visible y tangible pertenecen al mismo mundo. (Merleau-Ponty, 1997, p. 177)

Mas aún, hay recuperación doble y cruzada de lo visible en lo tangible y de lo tangible en lo visible; las dos cartas son completas y, sin embargo, no se confunden. Las dos partes son partes totales, pero no son superponibles (Merleau-Ponty, 1997, p. 177). Por eso podemos decir que "la visión es palpación por la mirada" (Merleau-Ponty, 1997, p. 177) y "relación de superposición" entre lo visible y lo tangible: "lo visible no es un cero de tangible, lo tangible no es un cero de visibilidad (relación de superposición)" (Merleau-Ponty, 1997, p. 178n).

Esta, y no otra, es la concepción merleau-pontiana de la carne: se trata de "esta Visibilidad, esta generalidad de lo Sensible en sí, este anonimato innato de Mí mismo al que llamamos carne" (Merleau-Ponty, 1997, p. 183). Si mis dos manos tocan las mismas cosas, es porque son "las manos de un mismo cuerpo". Incluso si cada una tiene su propia experiencia táctil, ellas interactúan con "un solo tangible": entre una y otra existe una relación especial, a través del espacio corporal, que hace de ambas un solo órgano de experiencia y de mis dos ojos, canales de una sola visión "ciclópea" (Merleau-Ponty, 1997, p. 186).

De modo que es preciso renunciar "a la bifurcación de la 'conciencia de' y del objeto, admitiendo que mi cuerpo sinérgico no es objeto, [...] que 'mi conciencia' no es la unidad sintética, increada, centrífuga, de una multitud de 'conciencias de..."' (Merleau-Ponty, 1997, p. 186). Por el contrario, mi cuerpo y el de todos los otros son "un Sintiente en general frente a un Sensible en general" (Merleau-Ponty, 1997, p. 187). Así como hay una sinergia al interior de cada uno, hay una sinergia entre diferentes organismos (MerleauPonty, 1997, p. 187). Es preciso, entonces, dejar de definir el sentir como 
"la pertenencia de una misma "conciencia'," y comprenderla "como retorno a sí de lo visible, adherencia carnal del sintiente al sentido y de lo sentido al sintiente", como recubrimiento y fisión, identidad y diferencia que "hace nacer en un rayo de luz natural que ilumina toda carne y no sólo la mía" (Merleau-Ponty, 1997, p. 187).

Por lo dicho, el alter ego no es aquí un problema "porque no soy yo quien ve, ni él quien ve" sino que "una visibilidad anónima nos habita a ambos, una visión en general" (Merleau-Ponty, 1997, p. 187). Merleau-Ponty habla de un "ser intercorporal, un dominio presuntivo de lo visible y de lo tangible" en el cual tanto el círculo de lo tocado y del tocante como el círculo de lo visible y del vidente, en sus relaciones recíprocas, inscriben lo tocante en lo visible, y lo vidente en lo tangible, propagando sus intercambios a todos los cuerpos (Merleau-Ponty, 1997, p. 188). En virtud de ello, cabe hablar de una reversibilidad del vidente y lo visible, del tocante y lo tocado; "de una reversibilidad siempre inminente y jamás realizada de hecho" puesto que nunca se logra una plena coincidencia entre ambos términos, lo que "implica un retorno a sí incesante que no se sabría resolver en un estado definitivo" (Alloa, 2008, p. 79). En efecto,

mi mano izquierda siempre está a punto de tocar mi mano derecha tocando las cosas, pero no alcanzo nunca la coincidencia; ella se eclipsa en el momento de producirse, y es siempre una entre dos cosas; o de verdad mi mano derecha pasa al rango de tocada, pero entonces su toma en el mundo se interrumpe, o bien ella la conserva, pero entonces es que no la toco de verdad, de ella, de mi mano izquierda solo palpo la envoltura exterior. Igualmente, no me escucho como escucho a los otros, la existencia sonora de mi voz para mí está por así decirlo mal desplegada; es sobre todo un eco de su existencia articular, ella vibra a través de mi cabeza más bien que fuera. (Merleau-Ponty, 1997, p. 194)

Esta circularidad en que una mano toca a la otra, y en que los movimientos exploradores y la experiencia táctil del cuerpo se mueven en el mundo, es caracterizada por Merleau-Ponty como la "metamorfosis de una de las experiencias en la otra" tal que "la bisagra entre ellas, sólida, inquebrantable" no me queda "irremediablemente oculta"; como si el "hiatus entre mi mano derecha tocada y mi mano derecha tocante, entre mi voz escuchada y mi voz articulada, entre un momento de mi vida táctil y el siguiente" no es "un vacío ontológico, un no-ser" gracias a que "es abarcado por el ser total de mi cuerpo, y por el del mundo" (Merleau-Ponty, 1997, pp. 194-195). 


\section{La crítica henriana}

El MARCo GENERAL DE LA CRÍTICA HENRIANA a Merleau-Ponty es su recuperación de Husserl. En efecto, Henry atribuye el hecho de que el cuerpo merleaupontiano sea "inmediatamente intencional" a que la subjetividad en Husserl (en la cual Merleau-Ponty se basa) ya "era intencional" (Henry, 2004, p. 305). De ahí que, si bien tiene el mérito de haber descubierto un cuerpo subjetivo, tiene también el demérito de haberlo concebido como "un cuerpo subjetivo intencional" y, por ello, de haber dejado "en la sombra una dimensión de otro orden" como la "dimensión patética", puesto que "nuestra corporeidad es fundamentalmente patética" (Henry, 2004, p. 305) y, por ende, inmanente. Así, es "la interioridad absoluta del esfuerzo a sus propios poderes" lo que "distingue el cuerpo orgánico de M. Henry del cuerpo propio husserliano" (Lipsitz, 2004, p. 75).

Más allá de estas consideraciones generales, Henry despliega dos series de argumentos detallados. Por un lado, en La esencia de la manifestación, el primer Henry (2003, p. 292/470) le objeta al primer Merleau-Ponty -el de Fenomenología de la percepción- su recaída en el dualismo; por otro lado, el último Henry critica al último Merleau-Ponty, cuestionando -en Encarnación - la fallida superación de la oposición entre el cuerpo sintiente y el cuerpo sentido mediante la absolutización de lo Sensible en Lo visible y lo invisible.

La primera de estas críticas se dirige contra la concepción gradualista de la realidad en Merleau-Ponty. Tajante, Herny (2003, p. 711) ${ }^{1}$ sostiene: no se puede decir que haya "grados de realidad en nosotros como hay 'reflejos', 'fantasmas' y 'cosas' fuera de nosotros". Por el contrario, habría que decir que el error y la ilusión concernientes al ser del sentimiento "no son reductibles ni comparables a los errores y las ilusiones que intervienen en el dominio donde el pensamiento está en sí” (Henry, 2003, p. 758)². Por ello,

\footnotetext{
${ }^{1}$ Véase también Henry, 2003, p. 799.

${ }^{2}$ Respecto de esta cuestión, la posición henriana es que no existen sentimientos falsos sino sentimientos mal comprendidos: "la ilusión o el error no conciernen jamás al sentimiento de sí-mismo y jamás le es interior [...] La ilusión o el error siempre se encuentra fuera del sentimiento, en la interpretación que de él se da el pensamiento [...] no hay mentira respecto de la afectividad y el sentimiento es lo menos ambiguo que hay" (Henry, 2003, p. 710).
} 
"la determinación ontológica fundamental del sentimiento como contenido inmanente y, recíprocamente, de todo contenido inmanente como afectivo" -es decir, "la determinación ontológica fundamental de la afectividad como constituyente de la esencia de la inmanencia y como idéntica a ella" (Henry, 2003, p. 758)- es irreductible a la determinación ontológica de la trascendencia.

En definitiva, lo que Henry encuentra reprobable en Merleau-Ponty es su rechazo a la estructura de la inmanencia y la ipseidad de la vida. Por eso considera ambiguo su posicionamiento, dado que el elemento oscuro es condición de la objetividad. Así, Henry se alza contra el olvido por parte de la conciencia de sus propios fenómenos y del cuerpo subjetivo, recordando que es la inmanencia la que funda la "oscuridad" en la que se extravía Merleau-Ponty a consecuencia de concebir, equivocadamente, la luz como luz exterior (Henry, 2003, p. 488-496).

Hemos dicho que Henry continúa su crítica a Merleau-Ponty en Encarnación. Según expone allí, en Lo visible y lo invisible la recaída en el dualismo cobra la forma de una fallida superación de la oposición entre el cuerpo sintiente y el cuerpo sentido mediante la absolutización de lo Sensible. Este intento es objeto de una concentrada objeción en el $\S 21$ de la mencionada obra, donde Henry considera objetable que, al hablar de una carne que no es la carne de nadie sino la carne del mundo ${ }^{3}$, Merleau-Ponty no haga más que conducir a paradojas que la despojan de todo aquello que le es propio. Así, por ejemplo, la descripción de las manos que se tocan en Lo visible y lo invisible, mostraría los absurdos que suscita esta perspectiva, los cuales tendrían su origen en la oposición del cuerpo constituyente y el cuerpo constituido y en la idea equivocada de que su relación es reversible (Henry, 2000, p. 152).

Con ello, Henry considera que Merleau-Ponty descalifica de un modo absurdo la estructura opositiva de lo constituyente y lo constituido

\footnotetext{
${ }^{3}$ Recuérdese que estas palabras fueron dichas por Merleau-Ponty en el marco de su última obra, en procura de una "fenomenología integral". No es, entonces, una extensión de la fenomenología sino una incursión en aquello que, desde nuestra experiencia, la excede. Por eso, el objeto de Lo visible y lo invisible no es simplemente el cuerpo sino ante todo el ser. De ahí que -tal como el mismo Henry lo señala-Merleau-Ponty hable allí de "ontogénesis" y no de constitución en sentido fenomenológico (Henry, 2000, p. 166).
} 
porque, subrepticia e ilegítimamente, extiende al mundo entero la relación tocante/tocado característica del cuerpo propio y que se produce solo en él. Así, estima que la mano que toca no es solo una parte tocada del cuerpo propio sino también una parte del mundo en general y que, por lo tanto, es homogénea con él. Al insertarse en el mundo, el poder transcendental de sentir testimonia su posibilidad permanente de devenir cosa entre las cosas; a la inversa, cuando la mano tocada, sita en el mundo, se convierte en la mano que toca, comparte con el mundo entero su condición (instalada por ella misma en su nuevo registro) de cuerpo transcendental, constituyente y constituido.

De esta doble posibilidad inherente al cuerpo trascendental de hacerse mundo - de convertirse en tocado, de tocante que era- y del mundo de convertirse en cuerpo trascendental -tocante, de tocado que era-resulta el Sensible merleau-pontiano: Sintiente/sentido, Tocante/tangible, Vidente/ visible, todo a la vez y de manera uniforme, el entrelazo, esta entidad tan ecléctica como inconcebible que pretende definir la única realidad, la del mundo -aparecer y contenido confundidos-, el mundo sensible, la carne del mundo. (Henry, 2000, pp. 165-166)

Vidente y visible no son dos momentos homogéneos que pudieran encarnarse uno en otro y ambos enrollarse en torno del Vidente "sino un solo [momento], el de la realidad, de manera que, inicialmente, el ser vidente, lo visible, y el ser-visible del Vidente no serían más que uno" (Henry, 2000, p. 166). De modo que, "entre tocar y ser tocado no existe la disimetría que afecta los análisis de Merleau-Ponty" pues, en Henry, ambos "no se oponen sino que son pensados como modalidades simultáneas" del yo puedo (Lipsitz, 2004, p. 78).

\section{La concepción henriana de la carne}

Al Destituir al tACTO DE SU estatuto PRIVILEGIAdo en la constitución del cuerpo propio, Henry se aparta "de manera decisiva" de los análisis de Husserl y Merleau-Ponty (Laoureux, 2005, p. 152). Contra ellos afirma -como una de las tesis decisivas de la fenomenología de la vida- que el comienzo de nuestra experiencia no es la intencionalidad sino las impresiones de la carne (Henry, 2000, pp. 226-227). Este análisis conduce a una aparente paradoja, que el mismo Henry pone de relieve al decir que "el 'tocante' originario -el 'ser tocado' - no es tocado por el sentido del tacto, por el 'tocante', entendido como el ejercicio de este sentido" (Henry, 2000, p. 228). En definitiva, es la 
carne la que actúa sobre el cuerpo cósico. Lo que es tocado en mí por mí en tanto que cuerpo cósico "no lo es, por lo tanto, más que en este despliegue de los poderes de mi carne" (Henry, 2000, p. 229). Tanto el "tocante" como lo "tocado"-el hecho de ser tocado- pertenecen solo a ella. Así, "la posibilidad de 'ser-tocado' reproduce la de ser 'tocante' al punto de serle idéntica. [Por eso], mi cuerpo cósico no es más tocante que tocado" (Henry, 2000, p. 229). Mi cuerpo cósico objetivo no siente nada, "no es más que la aparición exterior del contenido resistente alcanzado interiormente por mi carne como el límite de su poder. [Solo a mi carne pertenecen] el ser y el poder de ser tocados. Es la misma carne originaria la que es tocante y tocada al mismo tiempo" (Henry, 2000, p. 230). Por eso, es inexacto decir como Merleau-Ponty

que, cuando mi mano derecha toca mi mano izquierda se deja, al contrario, tocar por aquélla, abandona a la vez su habilidad, su condición de tocante para encontrarse absorbida en el tocar comprendido en el sentido de un tangible cualquiera, de un sensible cualquiera análogo a todos los cuerpos cósicos del universo. La verdad es todo lo contrario: cuando mi mano tocante se deja tocar por la otra mano, deviene una mano tocada, conserva su condición de carne originaria, esta auto-impresionalidad que puede sólo ser 'impresionada', 'tocada' por lo que sea. (Henry, 2000, p. 230)

De este modo, si bien "actividad y pasividad son dos modalidades fenomenológicas diferentes y opuestas, $[\ldots]$ son dos modalidades de una misma carne, su estatuto fenomenológico es el mismo, precisamente, el de esta carne" (Henry 2000: 230) cuyo movimiento es, como sostiene Lipsitz (2004, p. 74), "un automovimiento que nunca actúa fuera de sí".

La carne, entonces, es aquello que, al experimentarse -sufrirse, padecerse, soportarse a sí misma, gozar de sí-, es susceptible de sentir el cuerpo exterior, tocarlo y ser tocado por él (Henry, 2000, p. 10); sin embargo, ella se define por aquello de lo que se encuentra desprovisto todo cuerpo: de algún modo, es su contrario, puesto que ambos se oponen como el sentir y el no sentir (Henry, 2000, p. 11).

Por ello el cuerpo sensible no es el cuerpo sentido. El cuerpo sentido presupone el cuerpo sintiente del mismo modo que todo cuerpo visto presupone un poder de visión, lo mismo vale mutatis mutandis para el cuerpo sonoro, el cuerpo tocado y el palpado (Henry, 2000, p. 145-146):

somos reenviados ineluctablemente de un cuerpo sensible mundano, objeto del mundo, a un cuerpo totalmente de otro orden: un cuerpo 
trascendental provisto de sus poderes fundamentales de ver, de sentir, de tocar, de escuchar, de mover y moverse, y definido por ellos. Cuerpo "trascendental" porque es condición de posibilidad del cuerpo sentido, mundano. Cuerpo sintiente y ya no sentido, donante y ya no donado, un cuerpo que dona el mundo y el conjunto de cuerpos sentidos en él. (Henry, 2000, p. 159)

Henry distingue, allí donde Merleau-Ponty no lo hacía, un cuerpo subjetivo y un cuerpo objetivo. El cuerpo subjetivo trascendental "dona y siente el cuerpo sentido y donado por él -todo cuerpo objetivo mundano-"“ (Henry, 2000, p. 159). Es el elemento inmanente de nuestro cuerpo. Es el corazón y el núcleo del cuerpo trascendente que pertenece a la naturaleza: "Este elemento me aparece precisamente como el corazón y el núcleo del cuerpo-objeto que veo o que puedo tocar. Lo que llamamos inmanencia ha devenido así la esencia misma de lo trascendente" (Henry, 2006, pp. 150151).

La separación entre los dos polos del arco intencional en el que permanecería encerrado Merleau-Ponty le permite a Henry explorar la inmanencia radical de una manera distinta, entendida como la esfera en la que se realiza la experiencia de la visión. Por esta razón, la significación última de la expresión "veo" no descansa en la representación de mi visión, sino en mi visión misma en tanto que experiencia radicalmente inmanente (Henry, 2006, p. 153).

En consecuencia, las determinaciones corporales como la vista son ciegas porque el cuerpo expresa un elemento heterogéneo respecto de la manifestación. Por eso Descartes sostiene que es el alma la que ve y no el ojo; por ello, también, los animales no ven aunque tengan ojos. Así, la reducción en Descartes separa el aparecer y lo que en este aparece como distinto del aparecer -esto es, como el aparecer del aparecer-.

De ahí que Henry considere que el cogito encuentra su formulación última en la proposición videre videor: "me parece que veo". En efecto, tras practicar la reducción radical, que atañe al cuerpo, Descartes argumenta: At certe videre videor, audire, calescere. Lo que pone de manifiesto esta epojé es la pura visión, abstraída de toda relación con los ojos del cuerpo: la visión pura en tanto fenómeno. "El ojo humano, tachado por la reducción y reconocido como incapaz de realizar la visión, se rinde a la luz trascendental que Descartes llama 'luz natural'”' (Henry, 1985, p. 26). 
Ahora bien, al decir que al menos nos parece que vemos, Descartes quiere decir no solo que el videre es heterogéneo respeto del videor, sino también que es el "fenómeno absoluto", siguiendo las palabras de Henry (1985, p. 27):

Videor no designa otra cosa. Videor designa la semejanza primitiva, la capacidad original de aparecer y de donarse en virtud de la cual la visión se manifiesta y se dona originalmente a nosotros, no importa qué sea de la credibilidad y de la veracidad que deba concedérsele en tanto que visión, sea lo que sea que vea o crea ver y de su ver mismo.

Descartes encuentra, entonces, en el sentir, la esencia original del aparecer y lo interpreta como el último fundamento. En el sentir, el pensamiento despliega el fulgor de su manifestación, exhibiéndose como lo que es:

sentimos nuestro pensamiento, sentimos que vemos, que escuchamos, que tenemos calor. Es este sentir primitivo, en tanto es lo que es, es este aparecer puro idéntico a sí mismo y al ser que justamente lo define. Siento que pienso, luego soy. Ver es pensar ver [...] pero pensar ver, es sentir que se ve. Videor, en videre videor, designa este sentir inmanente al ver y que hace de él un ver efectivo, un ver que se siente ver. (Henry, 1985, p. 29)

Así que el concepto de sentir se desdobla en un sentir trascendental que encuentra su esencia en el ek-stasis, y un sentir primitivo del pensamiento -el sentimus nos videre-, opuesto radicalmente a aquél y que, en el sentirse a sí mismo, da originalmente el pensamiento a sí mismo, siguiendo el planteamiento de Henry (1985, p. 29), el sentirse a sí mismo en que reside la esencia del pensamiento no es solo diferente al sentir que se apoya sobre el ek-stasis, lo excluye de sí y es esta exclusión la que formula el concepto de inmediatez. Pero el $e k$-stasis se esencia como una interioridad radical.

Por lo dicho, el ver sensible presupone, en tanto visión de lo que ve, el aparecer a sí de la visión. El doble uso del signo "ver" permite ilustrar esta dualidad ontológica, pues "designa tanto el ojo, o al menos una propiedad suya, cuanto la experiencia interna trascendental de la visión. Se dice: es el ojo el que ve" (Henry, 2006, p. 154). Así entendida, la visión no es un fenómeno localizado en el espacio pues, de ser así, una visión tal "no vería nada de nada" (Henry, 2006, p. 154). Por el contrario, la visión solo es posible "en tanto que experiencia interna trascendental, es decir, [en tanto] una modalidad de la vida absoluta" (Henry, 2006, p. 154). Por eso puede 
argumentarse que la corporeidad carnal es el alma en tanto "ipseidad viviente del cogito subjetivo y autoafectivo" (Kühn, 2009, p. 189).

\section{Equivocaciones y equívocos}

HASTA AQUÍ, ENTONCES, HEMOS PRESENTADO las principales críticas de Henry a la concepción merleau-pontiana del cuerpo propio y la carne. En breve, digamos que Henry objeta una recaída en el dualismo así como una fallida superación de la oposición entre cuerpo sintiente y cuerpo sentido, impugnando su concepción gradualista de la realidad solidaria de un inaceptable rechazo de la estructura de la inmanencia. Esto llevaría a Merleau-Ponty a hablar de una carne que no es la carne de nadie sino la del mundo, despojándola así de todo lo que le es propio.

¿Es acaso incontestable esta serie de cuestionamientos? Encontramos dos aspectos en los que la crítica henriana debe ser revisada: uno, de orden semántico (un equívoco); otro, de interpretación (a nuestro juicio, una equivocación). En lo que sigue, mostraremos las diversas acepciones que el término "carne" tiene en las obras de Merleau-Ponty y Henry, depurando a partir de este cotejo el fondo mismo de la argumentación. Después, indagaremos la significación del dualismo como marco interpretativo de la obra de Merleau-Ponty y sus implicaciones para la arquitectura de conjunto de la obra de Henry. Con ello buscaremos allanar el camino hacia un posible diálogo, más profundo y menos doctrinario, entre ambos filósofos.

En cuanto a los equívocos, preciso es reparar en que la tesitura merleaupontiana se basa en la distinción hecha por Husserl entre Körper (cuerpo objetivo) y Leib (cuerpo viviente), este último entendido en tanto que "carne" (según lo hemos mostrado en el apartado 1). Ahora bien, Henry considera que la distinción entre Leib y Körper es limitada porque únicamente toma en cuenta elementos constituidos, representados, visibles (Laoureux, 2005, p. 152). En este sentido, la oposición entre Leib y Körper no hace más que describir la estructura oposicional de la conciencia, sin alcanzar nunca a la subjetividad viviente, que es de otro orden. Este desacuerdo filosófico conlleva un desplazamiento semántico consistente en que Henry llama "cuerpo orgánico" a lo que la fenomenología no henriana denomina "carne" $\mathrm{y}$, en cambio, designa como "carne" al "cuerpo fenomenológico originario" (Mathias, 2013, p. 147). 
Puede interpretarse, entonces, que Henry usa el término "carne" en el sentido de lo que la lengua alemana denomina Fleisch (Kühn, 2009, p. 186), entendida como una cualidad propia del "vivir corporal" específica e irreductible a la contraposición entre Körper y Leib (Kühn, 2009, p. 189). Con esta sustitución de términos, nuestra corporeidad es restituida a la Vida originaria y a la autoafección que le es propia, a saber, la "Autoafección fuerte" (Dufour-Kowalska, 2006, p. 86). De modo que, aclarado este equívoco, se hace evidente que la confrontación de Henry con MerleauPonty no tiene como eje una concepción distinta de un mismo término, sino la recuperación de una dimensión negada por la fenomenología histórica, que es esa dimensión subjetiva trascendental previa a la oposición entre Leib y Körper, única merecedora en sentido estricto del nombre Fleisch. Es decir que Henry nos habla de algo que ni siquiera ha recibido un nombre en las descripciones husserlianas y merleau-pontianas de la carne. Por lo tanto, en vez de una caracterización errónea, Henry debería haber señalado una omisión de la carne en los análisis de Merleau-Ponty.

En cuanto a las equivocaciones, creemos que no es procedente tomar -como lo hace Henry- a la filosofía declaradamente monista del último Merleau-Ponty como una "recaída en el dualismo". Henry no sólo cree que esto es así, sino que además le asigna un lugar de relevancia en su crítica a Merleau-Ponty, quien habría sido llevado por el dualismo a describir la carne del mundo como la carne de nadie, como la negación de mi experiencia, como la anti carne, ya que el mundo (para Henry, no para Merleau-Ponty) jamás será una carne ${ }^{4}$. El dualismo es el punto de partida de la crítica henriana, no su objeto. La dualidad ya está supuesta en la oposición ipseidad-mundo, que no es introducida por Merleau-Ponty sino por Henry, quien desde ella interpreta (a su manera, absolutamente peculiar) el objeto de sus diatribas. En definitiva, lo que induce a Henry a ver en Merleau-Ponty un dualista es que este sigue los pasos de la interpretación husserliana, conducida al hilo de la intencionalidad. Siendo, entonces, una perspectiva intencionalista, confinada a una filosofía de la conciencia, ha de encontrarse entonces -la exigencia, de gran coherencia interna en la obra de Henry aunque también de cierta tosquedad para el diálogo con otras ontologías- atrapado por la estructura oposicional sujeto-objeto.

\footnotetext{
${ }^{4}$ También en esta dirección, Marion (2005b, p. 134) considera que "no hay carne en el mundo (el mundo se define justamente por su ausencia radical de carne)".
} 
Además de introducir cierta inflexibilidad para comprender otras filosofías desde las claves que ellas mismas instituyen y sin proyectar de un modo a veces violento el propio armazón categorial sobre aquellas, cuando de la carne se trata, el dualismo le hace pagar un alto precio a Henry en los términos de la formulación de su propia problemática. Al respecto, Barbaras señala con gran perspicacia que, al mantener el concepto de cuerpo para designar su aparición en la exterioridad (tal es el caso del "cuerpo objetivo"), $\mathrm{y}$ al afirmar que este objeto del mundo puede ser aprehendido como habitado por una vida, Henry entra en "contradicción frontal" con su propio análisis del aparecer, pues con ello estaría admitiendo involuntariamente un "aparecer específico de la vida en la exterioridad, del sujeto en el mundo" (Barbaras, 2008 , p. 36). Habría en Henry, entonces, una tensión entre una inspiración metafísica dualista que afirma una duplicidad fundamental del aparecer y sitúa la vida del lado de la inmanencia pura, y una exigencia descriptiva que lo conduce a reconocer que algo de la vida se da en el mundo, haciendo peligrar así las bases mismas de su fenomenología (Barbaras, 2008, p. 37).

De todos modos, nada ganaremos con poner en evidencia algunas contradicciones en la crítica henriana a Merleau-Ponty, ni con señalar el carácter colérico y visceral de sus embates. Lo que nos interesa es mostrar que la propuesta de Merleau-Ponty va más allá que lo considerado por Henry, y que ese plus tiene un carácter conciliador -no en vano ha quedado fuera del análisis, pues de lo contrario algunas expresiones deberían matizarse y las diferencias se relativizarían. Para ello, hemos de mostrar que una de las condiciones que Henry le impone a cualquier superación de los desvaríos que cree encontrar en Merleau-Ponty, es pensar la emergencia de esta "argamasa", que es el enlace "del espacio y del espíritu" a partir de la "espiritualidad" cartesiana (Henry, 2006, p. 59). En lo que sigue, nos proponemos exponer que este último movimiento, rectificatorio de algunos de los desvíos señalados, se encuentra en Merleau-Ponty, a pesar de que Henry no espere hallarlo ahí.

\section{Acortando distancias}

MerLEau-Ponty le Dedica a la espiritualidad cartesiana los cursos de abril y mayo de 1961, reparando en la importancia de "la visión de la visión" en Descartes (Merleau-Ponty, 1996, p. 232), a pesar de que cuestiona este "comienzo cartesiano" en tanto que "positivismo de la visión del espíritu" (Merleau-Ponty, 1996, p. 233). 
A fin de rescatar los hallazgos cartesianos sin que ellos se vean malogrados por dicho positivismo, Merleau-Ponty interpreta esta visión como una "modificación de la luz natural" tal que ya no se trata de un "ver como separado, distintivamente, en virtud de una organización como la del cuerpo [sino de un ver con] los ojos del espíritu" (Merleau-Ponty, 1996, pp. 262-263), un ver que "no es ver" (Merleau-Ponty, 1996, p. 268) sino "pensamiento de ver y de sentir" (Merleau-Ponty, 1996, p. 244).

En esta identidad de la visión y lo visto en la luz -es decir, en lo visto "en mí, luz humana" (Merleau-Ponty, 1996, p. 239)-, "el cogito no separa de mí el sentir, el pensamiento está completo en el sentir" (Merleau-Ponty, 1996, p. 266); de modo que, si bien conocer es ver, aquí, "lo que se ve y el que ve son uno, es la luz revelándose a sí misma al mismo tiempo que a los objetos" (Merleau-Ponty, 1996, p. 235). Es decir que, "para este entendimiento existente", "nada puede ser 'separado de sí" " (Merleau-Ponty, 1996, pp. 264-265). Por eso -interpreta Merleau-Ponty- (1996, p. 244) que "las Meditaciones... le agregan a la certeza, la certeza de la certeza"; más aún, la "certeza de la presentación, de la aparición a... del testimonio interior, del 'conocimiento interior' no tético, de la no-disimulación de mí a mí y de todas las cosas que son inmediatamente verdad" (Merleau-Ponty, 1996, pp. 266-267).

¿Qué ha quedado del dualismo, de la oposición entre el cuerpo sintiente y el cuerpo sentido, de la carne entendida en una acepción mundana?, ¿qué ha quedado de la caracterización henriana del pensamiento de MerleauPonty en lo que dejan traslucir estos cursos? ¿Qué dualismo entrever en esta identidad de la visión y lo visto, en un cogito que no se separa de mi sentir y un pensamiento que está completo en el sentir? ¿Qué distancia establecer en este conocer que es un ver, que es uno con lo que se ve, en esa luz revelándose a sí misma al tiempo que ve los objetos? ¿Por qué este testimonio interior del conocimiento no tético habría de estar en los antípodas de una fenomenología de la inmanencia y la afectividad?

Tal vez el Descartes de Merleau-Ponty no llegue a echar por tierra la totalidad de la crítica henriana pero al menos recupera -eso creemos- un terreno fecundo para una lectura comparada que, sin enfatizar unilateralmente las profundas diferencias que puedan separar a Merleau-Ponty de Henry, al menos sepa encontrar algunas significativas convergencias entre ambos. 
Particularmente, creemos que la interpretación merleau-pontiana de Descartes se aproxima a la tesitura henriana en que logra ir más allá, en su radicalidad, a esa dimensión previa al despliegue de la estructura oposicional de la conciencia donde el cuerpo se encuentra -tal como lo muestra Henry-atrapado en la dualidad de lo constituyente y lo constituido, en la dimensión trascendental que es la carne henriana, donde el sintiente se revela inmediatamente a sí mismo como sentido y que no es otra cosa que el espíritu cartesiano. Allí, en esa luz interior, también Merleau-Ponty llega a admitir algo de lo que magistralmente nos muestra Henry: que no es el ojo sino la carne quien ve (Henry, 2000, p. 287).

\section{Conclusiones}

A MODO DE SíNTESIS DE LA BELIGERANTE RELACIÓN que ha sostenido Henry con Merleau-Ponty, queremos recuperar en nuestras conclusiones dos cuestiones: por un lado, Henry no advierte en qué medida la idea de "sobrereflexión" aleja a Merleau-Ponty de la intencionalidad propia de la "fenomenología histórica"; por el otro, no percibe en toda su dimensión el valor que tienen para su adversario la inmanencia y el legado cartesiano.

En cuanto a la sobrereflexión, Merleau-Ponty muestra en Lo visible y lo invisible que ella articula los dos polos que el dualismo escindía mediante un entrelazo que se da, todo entero, en una experiencia y no fuera de ella. Lejos de asignarle a mi experiencia el carácter propio de un mundo inerte, Lo visible y lo invisible generaliza la reflexividad de la experiencia, no su negación. ¿Acaso es tan distinto este Sintiente en general, de lo que en Henry aparece como la problemática de la comunidad? ${ }^{5}$ ¿No tienen algo en común los sintientes, como lo tienen los "vivientes" henrianos? Ciertamente. Creemos que la generalidad del Sintiente, que Henry interpreta como si no fuese nadie, es, en tanto "carne del mundo" (expresión que escandaliza a Henry), lo que tenemos en común los "sintientes". Esta incomprensión va de la mano de la segunda, ya que lo que Henry no comprende es que todo (el mundo, el Ser, incluso) se da en una experiencia y, por ende, en la inmanencia -que no será la inmanencia de la vida henriana, ciertamente, pero sí al menos la de lo sensible, estopa de la cual todo está hecho.

\footnotetext{
${ }^{5} \mathrm{Al}$ respecto, véase: Henry, 1990, pp. 160-179; Lipsitz, 2004, pp. 53-56/70-72; Belvedere (2011).
} 
En lo que concierne a la inmanencia, entonces, es preciso reparar en que Merleau-Ponty se orienta hacia una tematización de la luz interior y la reversibilidad. Ciertamente, caracteriza esta esfera muy de otra manera que Henry, pero no puede decirse que la desconozca. También MerleauPonty es un cartesiano que encuentra en el espíritu, en la luz interior y en el sentimiento, lo que nos es propio.

De todos modos, tiene razón Henry en que se trata de una experiencia de la inmanencia distinta a la que sus propios análisis muestran. Queremos hacer notar aquí una diferencia, abismal, de la cual Merleau-Ponty está consciente, como si -por un extraño anacronismo- tuviera en mente las tesis henrianas al cuestionar lo que podríamos llamar "inmanentismo", por considerarlo oneroso:

Reducir la percepción al pensamiento de percibir, bajo pretexto de que solo la inmanencia es segura, es un seguro contra la duda cuyas primas son más onerosas que la pérdida por la cual nos ha de compensar: pues es renunciar a comprender el mundo efectivo y pasar a un tipo de certeza que jamás nos dará el "hay" del mundo. (Merleau-Ponty, 1997, pp. 58-59)

¿No basta, acaso, con esta sola cita para mostrar que, como dijimos al principio, la confrontación de Henry con Merleau-Ponty era inevitable? 


\section{Referencias}

AlloA, E. (2008). La Résistance du sensible. Merleau-Ponty critique de la transparence. Paris: Kimé.

Barbaras, R. (2008). Introduction à une phénoménologie de la vie. Paris: Vrin.

Barbaras, R. (1997). Merleau-Ponty. Paris: Ellipses.

Belvedere, C. (2011). Intersubjetividad y comunidad en la fenomenología material de Michel Henry. C. B. González \& M. L. Ballabeni. (Comps.), Experiencia e historia: identidad, comunidad, universalidad. Estudios de fenomenología y hermenéutica IV. Paraná: Círculo de Fenomenología y Hermenéutica de Santa Fe-Paraná.

Dufour-Kowalska, G. (2006). Le Corps subjectif: Incarnation et Révélation. Michel Henry. Pensée de la vie et culture contemporaine. Paris: Beauchesne.

García-Baró, M. (1999). Vida y mundo. La práctica de la fenomenología. Madrid: Editorial Trotta.

Henry, M. (2006). Philosophie et phénoménologie du corps. Paris: Presses Universitaires de France.

Henry, M. (2004). Phénoménologie de la vie. III. De l'art et du politique. Paris: Presses Universitaires de France.

Henry, M. (2003). L'Essence de la manifestation. Paris: Presses Universitaires de France.

Henry, M. (2000). Incarnation. Une philosophie de la chair. Paris: Seuil.

Henry, M. (1990). Phénoménologie Matérielle. Paris: Presses Universitaires de France.

Henry, M. (1985). Généalogie de la psychanalyse. Le commencement perdu. Paris: Presses Universitaires de France.

HuSSERL, E. (2004). Idées Directrices pour une phénoménologie et une philosophie phénoménologique pures. Livre seconde. Recherches phénoménologiques pour la constitution. Paris: Presses Universitaires de France.

KüHn, R. (2009). Corporéité et vie charnelle. Résultats et perspectives de la phénoménologie matérielle. J.-M. Brohm \& J. Leclercq (Eds.), Michel Henry. Paris: L' Âge d'Homme. 
Laoureux, S. (2005). L'Immanence à la limite. Recherches sur la phénoménologie de Michel Henry. Paris: Cerf.

Lipsitz, M. (2004). Eros y Nacimiento fuera de la ontología griega: Emmanuel Levinas y Michel Henry. Los Polvorines/Buenos Aires: Universidad Nacional de General Sarmiento/Prometeo.

Marion, J.-L. (2005a). Acerca de la donación. Una perspectiva fenomenológica. Buenos Aires: Jorge Baudino/Universidad Nacional de San Martín.

Marion, J.-L. (2005b). El fenómeno erótico. Seis meditaciones. Buenos Aires: Ediciones Literales.

Mathias, G. (2013). La Praxis Michel Henry : corps et esprit de la phénoménologie matérielle. Revue Internationale Michel Henry, 4. Louvain: Presses Universitaires de Louvain.

Merleau-Ponty, M. (1999). Phénoménologie de la perception. Paris: Gallimard.

Merleau-Ponty, M. (1997). Le Visible et l'invisible. Paris: Gallimard.

Merleau-Ponty, M. (1996). Notes de cours au Collège de France. 1958-1959 et 1960-1961. Paris: Gallimard. 